\title{
Editorial
}

\section{Artificial intelligence applications for Ophthalmology: Current status}

\author{
Chetan Rao', Rajiv Raman ${ }^{1}$ \\ ${ }^{1}$ Sri Bhagwan Mahavir Vitreoretinal services, Sankara Nethralaya, Chennai
}

What is intelligence? It is the ability to learn, reason, problem-solving and adapt to the environment. It is a general ability to use multiple cognitive functions like perception, memory, logic, language and planning. This is what a human mind is capable of, however not all humans think alike or use their cognitive powers to solve problems efficiently and decision making is subjective. This is where the research in artificial intelligence has made giant strides and aims to augment the human abilities in solving problems at a large scale with objectivity, speed and accuracy.

Artificial intelligence (AI) is a branch of computer science that was conceptualized in the 1950s with the aim to use computers and complex algorithms to perform functions that can simulate the functioning of the human mind.

A subset field of AI is "Machine learning" that uses various statistical techniques and computer algorithms to learn automatically by experience alone after training on datasets, under supervision or without it.

Inspired by the human neural network of the brain,artificial neural networks (ANN) were developed that could intelligently learn to analyse large data-sets using algorithms. These networks had multiple processing units interlinked to one another like the brain neurons to analyse the input data and predict the most probable outcome for which it was designed. Deep learning is a special kind of neural network that can learn a long chain of causal links between layers of networks to finally give an output. It is based on learning features from the data by processing a large amount of data and extracting meaningful patterns from them (Krizhevsky et al, 2012). A special type of neural network called the convolutional neural network (CNN) learns to perform a task by repetitive analysis of data with feed-back looping and thereby self- correcting and auto-tuning its results (Browne et al, 2003). The network algorithms typically analyses human expert-labelled, image dataset and adjusts its parameters till it decreases the error in diagnosis compared to human graders by repeating the process for every image multiple times. Once the algorithms achieves high level of accuracy, it is ready for working on unknown images. The deep neural networks are better than human in detecting subtle changes, patterns or peculiarities in images (LeCun et al, 2015).

The CNN architecture of deep learning is most suitable for analysing image data sets. They are composed of a special type of layer that apply a mathematical filtering operation called convolution that makes the individual neuron to process data only for its receptive field. As the input image is processed through successive such layers, parts of the image called motifs (feature detector) are defined and learnt. By repeated training on raw image data, the detectors or the neurons using these feature detectors 
learn to recognize these specific features to solve a particular image recognition task. By training on large annotated datasets, the CNNs start recognizing visual patterns (Nebauer C, 1998).

\section{Development of AI models for ophthalmic image modalities}

To develop an AI model, large amounts of raw imaging data such as colour fundus image, optical coherence tomography, ocular ultrasound B-scan, slit lamp image and visual field is needed for training ( $\mathrm{Lu} \mathrm{W}$ et al, 2018). The data needs to be preprocessed to get high quality images to optimize the learning process. All data from different sources needs to be standardized and most relevant features of the raw image is selected and extracted to improve the learning process.

The next step is to train the AI to process the images by defining the parameters of the model for example in a model to train the AI to diagnose diabetic maculopathy in a fundus image, labelled datasets that have features of diabetic maculopathy as recognised by a retina specialist are used. The model is then validated on another dataset to improve its accuracy and then tested on another dataset to evaluate the final performance of the trained model. The model's performance is cross validated to optimize its accuracy in predicting the outcome. Finally a receiver operating characteristic curve ( ROC), a statistical tool is used to depict the performance of the algorithm. The area under the ROC (AUC) is the most used evaluation metric for quantitative assessment of an AI diagnosis model. Typically an effective model will have values ranging from 0.5 to 1 ; the higher the value of AUC, the better is the performance of the model.

\section{Diabetic retinopathy}

The earliest application of AI in ophthalmology has been in diagnosing and grading diabetic retinopathy. Gulshan et al (2016) showed the utility of deep learning in evaluating referable diabetic retinopathy defined as moderate or worse diabetic retinopathy or macular edema wherein their algorithm evaluated 2 sets of image, one with 9963 images and the other with 1748 images and achieved $90.3 \%$ and 87\% sensitivity and $98.1 \%$ and $98.5 \%$ specificity respectively. The application of the Deep learning algorithm as a screening program in a nationwide study by Raumviboonsuk et al (2019) in Thailand showed higher sensitivity than human graders ( 0.97 vs 0.74$)$ with a slightly lower specificity ( 0.96 vs 0.98$)$. The outcomes of these and several other AI models have shown the potential for using deep learning algorithms in screening programs for detecting a high prevalence disease such as diabetic retinopathy and prevent morbidity by instituting early intervention.

\section{Age related macular degeneration}

AI models have been developed to detect and grade AMD using OCT with a sensitivity of $98.2 \%$ and specificity of $91.2 \%$ (Venhuizen F.G. et al, 2017) and from colour fundus images with accuracy between $88.4 \%$ (SD, $0.7 \%$ ) and $91.6 \%$ (SD, $0.1 \%$ ) Burlina et al (2017). Researchers have also used deep learning to predict progression of AMD and visual acuity after anti-vegf injections. The AI model may be a more cost-effective technique to screen patients for population based studies and maybe help clinicians to diagnose high risk AMD. 


\section{Retinopathy of prematurity}

Deep learning models (Mazzaferri et al, 2018) have been used to quantify disease process in Oxygen induced retinopathy (OIR) rodent models. Ataer- Cansizoglu et al (2105) developed an algorithm to diagnose plus disease with accuracy of $95 \%$. Machine learning approach can improve the inter-grader variability between clinicians and improve the screening of ROP.

\section{Glaucoma}

Using AI models several groups have shown high level of sensitivity and specificity in diagnosing glaucomatic optic neuropathy on fundus photography and by using an AI with a combination of IOP, corneal thickness, OCT and visual field data were able to differentiate glaucoma from healthy eyes (Kim et al, 2017).

The use of AI in ophthalmology is expanding with newer models being developed with multiple input data being used to predict diagnosis, treatment outcomes and research questions with greater accuracy. However the AI models are heavily dependent on large data sets and therefore that limits the application of the models in rare disease entities.

The paucity of physicians and the increase in disease prevalence is a gap that the AI models can bridge for delivering early and accurate diagnoses that can potentially improve health care. Ethical issues arise whenever a decision making process is done by a machine versus a human expert with regards to patient care. At best the AI models can augment the physicians but cannot replace them at present. Also, how a AI model reached a conclusion is still difficult to know as it self learns and lends itself to scrutiny in cases of misdiagnosis or bad treatment outcome. Researches have made some headway by using heatmaps that can be analysed to know the decision making process by the AI algorithm. Artificial intelligence is here to stay. It has been used historically in engineering applications but now artificial intelligence in health care is making rapid strides and will become the physicians best friend in clinic.

\section{References:}

Ataer-Cansizoglu E, Bolon-Canedo V, Campbell JP, Bozkurt A, Erdogmus D, Kalpathy-Cramer J et al (2015). Computer-Based Image Analysis for Plus Disease Diagnosis in Retinopathy of Prematurity: Performance of the "i-ROP" System and Image Features Associated With Expert Diagnosis. Transl Vis Sci Technol. Nov;4(6):5.

Browne M, Ghidary SS (2003). Convolutional neural networks for image processing: an application in robot vision. In: Gedeon T (Tom) D, Fung LCC (eds). AI 2003: Advances in Artificial Intelligence. Springer Berlin Heidelberg, pp 641-52.

Burlina PM, Joshi N, Pekala M, Pacheco KD, Freund DE, Bressler NM (2017). Automated Grading of Age-Related Macular Degeneration From Color Fundus Images Using Deep Convolutional Neural Networks. JAMA Ophthalmology. Nov 1;135(11):1170.

Gulshan V, Peng L, Coram M, Stumpe MC, Wu D, Narayanaswamy A et al (2016). Development and Validation of a Deep Learning Algorithm for Detection of 
Diabetic Retinopathy in Retinal Fundus Photographs. JAMA. 13;316(22):2402-10.

Kim SJ, Cho KJ, Oh S (2017). Development of machine learning models for diagnosis of glaucoma. Liu B, editor. PLOS ONE. May 23;12(5):e0177726.

Krizhevsky A, Sutskever I, Hinton GE (2012). Imagenet classification with deep convolutional neural networks. In: Pereira F, Burges CJC, Bottou L, Weinberger KQ (eds). Advances in Neural Information Processing Systems 25. Curran Associates, Inc., Redhook, pp 1097-105.

LeCun Y, Bengio Y, Hinton G (2015). Deep learning. Nature. May 28; 521(7553):436-44.

Nebauer C (1998). Evaluation of convolutional neural networks for visual recognition. IEEE Trans Neural Netw; 9(4):685-96.

Lu W, Tong Y, Yu Y, Xing Y, Chen C, Shen Y (2018). Applications of Artificial Intelligence in Ophthalmology: General Overview. Journal of Ophthalmology. Nov 19;2018:1-15.

Mazzaferri J, Larrivée B, Cakir B, Sapieha P, Costantino S (2018). A machine learning approach for automated assessment of retinal vasculature in the oxygen induced retinopathy model. Sci Rep. Mar 2;8(1):3916. doi: 10.1038/s41598-01822251-7

Raumviboonsuk P, Krause J, Chotcomwongse P, Sayres R, Raman R, Widner K et al (2019). Deep learning versus human graders for classifying diabetic retinopathy severity in a nationwide screening program. npj Digital Medicine [Internet]. 2019 Dec [cited 2019 May 11];2(1). Available from: http://www.nature.com/articles/s41746-019-0099-8.

Venhuizen FG, van Ginneken B, van Asten F, van Grinsven MJJP, Fauser S, Hoyng CB, et al (2017). Automated Staging of Age-Related Macular Degeneration Using Optical Coherence Tomography. Investigative Opthalmology \& Visual Science. Apr 24;58(4):2318. 